\title{
AFFINE MANIFOLDS AND SOLVABLE GROUPS
}

BY D. FRIED, W. GOLDMAN AND M. W. HIRSCH

Let $M$ be a compact affine manifold. Thus $M$ has a distinguished atlas whose coordinate changes are locally in $\operatorname{Aff}(E)$, the group of affine automorphisms of Euclidean $n$-space $E$. Assume $M$ is connected and without boundary.

The universal covering $\widetilde{M}$ of $M$ has an affine immersion $D: \widetilde{M} \rightarrow E$ which is unique up to composition with elements of $\operatorname{Aff}(E)$. Corresponding to $D$ there is a homomorphism $\alpha: \pi \rightarrow \operatorname{Aff}(E)$, where $\pi$ is the group of deck transformations of $\widetilde{M}$, such that $D$ is equivariant for $\alpha$. Set $\alpha(\pi)=\Gamma$. Let $L: \operatorname{Aff}(E) \rightarrow$ $G L(E)$ be the natural map.

THEOREM 1. If $\Gamma$ is nilpotent the following are equivalent:

(a) $M$ is complete, i.e. $D: \widetilde{M} \rightarrow E$ is bijective;

(b) $D$ is surjective;

(c) no proper affine subspace of $E$ is invariant under $\Gamma$;

(d) $L(\Gamma)$ is unipotent;

(e) $M$ has parallel volume, i.e. $L(\Gamma) \subset S L(E)$;

(f) $M$ is affinely isomorphic to $\Gamma \backslash G$ where $G$ is a connected Lie group with a left-invariant affine structure and $\Gamma \subset G$ is a discrete subgroup;

(g) each de Rham cohomology class of $M$ is represented by a differential form whose components in affine charts are polynomials.

For abelian $\Gamma$ the equivalence of (a), (d), and (e) is due to J. Smillie. We conjecture that (a), (b), (e), and (g) are equivalent even without nilpotence (if $M$ is orientable). In general (a) $\Rightarrow$ (c) and (e) $\Rightarrow(c)$; but (c) $\nRightarrow(a)$ even for $\Gamma$ solvable and $M$ three-dimensional.

THEOREM 2. The following are equivalent:

(i) $M$ is finitely covered by a complete affine nilmanifold $M_{1}$ (i.e. conditions (a) through (g) of Theorem 1 hold for $M_{1}$ );

(ii) all eigenvalues of elements of $L(\Gamma)$ have norm 1 ;

(iii) $M$ has a Riemannian metric whose coefficients in affine charts are polynomials.

L. Auslander has conjectured that if $M$ is complete then $\pi=\Gamma=\pi_{1}(M)$ is virtually solvable (i.e. contains a solvable subgroup of finite index); see [M] for discussion. This conjecture is true in dimension three (see [FG]).

Received by the editors May 29, 1980 and, in revised form, July 10, 1980.

1980 Mathematics Subject Classification. Primary 57N15, 22 E25.

๑ 1980 American Mathematical Society 
THEOREM 3. If $\pi$ is virtually solvable and $M$ is complete then (e), (f), (g) of Theorem 1 hold. If $\alpha: \pi \rightarrow \Gamma$ factors through a virtually polycyclic group of rank $\leqslant \operatorname{dim} M$ and $M$ has parallel volume, then $M$ is complete. In particular if $M$ is finitely covered by a manifold homeomorphic to a solvmanifold then parallel volume is equivalent to completeness.

We briefly indicate the proof of Theorem 1 .

(a) $\Rightarrow$ (c). This holds for any compact complete $M$. If $F \subset E$ is a $\Gamma$-invariant affine subspace then both $E / \Gamma$ and $F / \Gamma$ are Eilenberg-Mac Lane spaces of type $K(\pi, 1)$. Since they are compact manifolds their dimensions are equal; thus $F=E$.

(e) $\Rightarrow$ (c). This holds for all compact $M$. The linear holonomy $\rho=L \circ \alpha$ : $\pi \rightarrow G L(E)$ determines a $\pi$-module $E_{\rho}$. Let $u$ : $\pi \rightarrow E$ send $g \in \pi$ into the translational part of $\alpha(g)$. Then $u$ is a crossed homomorphism whose cohomology class $c_{M} \in H^{1}\left(\pi ; E_{\rho}\right)$ depends only on $M$. The $n$th exterior power $\Lambda^{n} c_{M} \in$ $H^{n}(\pi ; \mathbf{R})$ vanishes if and only if $M$ does not have parallel volume. On the other hand $c_{M}$ can be expressed in de Rham cohomology of $M$ with coefficients in $E$ twisted by $\rho$. Suppose $F \subset E$ is $\Gamma$-invariant. We may assume $F$ is a linear $\rho$-invariant subspace. Then $c_{M}$ comes from $H^{1}\left(\pi ; F_{\rho}\right)$. If $\operatorname{dim} F<n=\operatorname{dim} E$ then $\Lambda^{n} c_{M}$ comes from $H^{1}\left(\pi ; \Lambda^{n} F\right)=0$.

From now on assume $\Gamma$ is nilpotent.

(c) $\Rightarrow$ (d). Let $E_{U} \subset E$ be the maximal unipotent submodule. Then $H^{0}\left(\pi ; E / E_{U}\right)=0$, and nilpotent implies $H^{1}\left(\pi ; E / E_{U}\right)=0$ (Hirsch [H]). This means some coset of $E_{U}$ is $\Gamma$-invariant.

(b) $\Rightarrow$ (d). Suppose $E_{U} \neq E$. Some coset of $E_{U}$ is $\Gamma$-invariant; we may assume $E_{U}$ is $\Gamma$-invariant. There is a unique $L(\Gamma)$-invariant splitting $E=E_{U} \oplus F$. Let $M_{1}=p\left(D^{-1} E_{U}\right)$ where $p: \widetilde{M} \rightarrow M$ is the projection. Then $M_{1}$ is a compact affine manifold with unipotent holonomy, hence complete. Let $Y$ be the vector field on $\widetilde{M}$ which is $D$-related to the vector field $(x, y) \mapsto(0, y)$ on $E_{U} \oplus$ $F$. Then $Y$ covers a vector field on $M$, so $Y$ is completely integrable. Every component of $p^{-1} M_{1}$ is a repellor for $Y$. One uses these facts to prove that $M$ is complete; but this implies (c), and hence (d).

(d) $\Rightarrow$ (a). When $L(\Gamma)$ is unipotent there is a flag $E=E_{n} \supset \cdots \supset E_{0}=$ $\{0\}$ of $L(\Gamma)$-invariant linear subspaces with $L(\Gamma)$ acting trivially on each $E_{i} / E_{i-1}$. There are nested foliations $Z_{n}, \ldots, Z_{0}$ on $M$ covered by foliations $\widetilde{Z}_{i}$ on $\widetilde{M}$ such that $D$ relates $\widetilde{Z}_{i}$ to the linear foliation $E_{i}$ of $E$ whose leaves are cosets of $E_{i}$. For each $i$ there is a closed 1 -form $\widetilde{\omega}_{i}$ on ${\widetilde{Z_{i}}}_{i}$ which vanishes on $\widetilde{Z}_{i-1}$, related by $D$ to a constant 1-form on $E$ vanishing on $E_{i-1}$. There are completely integrable vector fields $X_{i}$ in $\widetilde{Z}_{i}$ with $\left\langle X_{i}, \omega_{i}\right\rangle=1$. Given any $p \in \widetilde{M}, x \in E$ one shows that the trajectory of $X_{n}$ through $p$ meets a point $p_{1}$ such that $D\left(p_{1}\right)$ is the leaf of $E_{n-1}$ through $x$. The trajectory of $X_{n-1}$ through $p_{1}$ stays in a leaf of $\widetilde{Z}_{n-1}$ and eventually meets a $p_{2}$ such that $D\left(p_{2}\right)$ is the leaf of $E_{n-2}$ through $x$, etc. In this 
way one proves that $D(\widetilde{M})$ contains a path from $D(p)$ to $x$. Hence $D$ is surjective. Injectivity is proved similarly.

(e) $\Rightarrow$ (d). If $E_{U} \neq E$ let $F \subset E$ be a complementary submodule to $E_{U}$. One shows that some element of $L(\Gamma)$ expands $F$, contradicting parallel volume.

(a) $\Rightarrow$ (b) and (d) $\Rightarrow$ (e) are obvious.

(a) $\Rightarrow$ (f). $G$ is the algebraic hull of $\Gamma$ in $\operatorname{Aff}(E)$.

(f) $\Rightarrow(\mathrm{g})$. By Nomizu's theorem [N] the cohomology of $M$ is represented by invariant forms on $G$; these turn out to be polynomial.

$(\mathrm{g}) \Rightarrow(\mathrm{e})$. If $L(\Gamma)$ is not unipotent then one proves there is no polynomial volume form.

\section{REFERENCES}

[F] D. Fried, Polynomials on affine manifolds (to appear).

[FG] D. Fried and W. Goldman, Three-dimensional affine crystallographic groups (in preparation).

[FGH] D. Fried, W. Goldman and M. Hirsch, Affine manifolds with nilpotent holonomy (to appear).

[G] W. Goldman, Two examples of affine manifolds, Pacific J. Math. (to appear).

[GH1] W. Goldman and M. Hirsch, Parallel characteristic classes of affine manifolds (in preparation).

[GH2] - A generalization of Bieberbach's theorem (to appear).

[GH3] - Polynomial forms on affine manifolds (in preparation).

[H] M. Hirsch, Flat manifolds and the cohomology of groups, Algebraic and Geometric Topology, Lecture Notes in Math., vol. 664, Springer-Verlag, Berlin and New York, 1977.

[M] J. Milnor, On fundamental groups of complete affinely flat manifolds, Adv. in Math. 25 (1977), 178-187.

[N] K. Nomizu, On the cohomology ring of compact homogeneous spaces of nilpotent Lie groups, Ann. of Math. (2) 59 (1954), 531-538.

[S] J. Smillie, Affinely flat manifolds, Doctoral dissertation, Univ. of Chicago, 1977.

DEPARTMENT OF MATHEMATICS, UNIVERSITY OF CALIFORNIA, SANTA CRUZ, CALIFORNIA 95064

DEPARTMENT OF MATHEMATICS, UNIVERSITY OF CALIFORNIA, BERKELEY, CALIFORNIA 94720 
